\title{
Optimized Genetic Algorithm (OGA) for Homogeneous WSNs
}

\author{
${ }^{1}$ Tripti Sharma, ${ }^{2}$ G. S Tomar, ${ }^{3}$ Radhika Gandhi, ${ }^{3}$ Srishti Taneja \\ and ${ }^{3}$ Kiran Agrawal \\ ${ }^{1}$ Deptt of IT, Maharaja Surajmal Institute of Technology, New Delhi-110058 \\ ${ }^{2}$ Machine Intelligence Research Lab, Gwalior \\ ${ }^{3}$ Maharaja Surajmal Institute of Technology, New Delhi \\ triptionline@yahoo.com,radhikagandhi@ymail.com,srishti2303@gmail.com, \\ kiran.agrawal13a@gmail.com
}

\begin{abstract}
A wireless sensor network (WSN) is a network consisting of wireless computing devices called sensors that sense environmental conditions like motion, sound, etc in an area. These networks collect the information from the environment and send it to the sink node. The main constraint in these networks is the energy of the nodes. As these sensors have limited battery life, routing protocol should be designed appropriately so that minimal energy is used. Long communication distances between the sensors and the sink in the WSN drain the energy of the sensors and reduce the lifetime of the network. Clustering reduces energy consumption. By clustering a sensor network we can help minimize the total communication distance, thus increasing the network lifetime. In this paper, we an algorithm based on LEACH (Low Energy Adaptive Clustering Hierarchy protocol) using genetic algorithm has been proposed in order to achieve increased lifetime of the network and energy efficiency in WSN. The genetic algorithm is used to select the cluster heads for the WSN and hence create the energy efficient clusters for transmission of data in the wireless sensor network. The fitness function proposed in this algorithm considers both the distance of the nodes from the sink and their energies. The simulation results show that the proposed protocol results in prolonged network lifetime and optimal energy consumption of the wireless sensor network.
\end{abstract}

Keywords: Wireless Sensor Network, LEACH, Genetic Algorithm, Fitness Function

\section{Introduction}

A wireless sensor network consists of a large number of sensor nodes that are deployed in environmental fields to serve various applications[1,2,3]. Facilitated with the ability of wireless communication and intelligent computation, these nodes become smart sensors which do not only perceive ambient physical parameters but also be able to process information, cooperate with each other and self-organize into the network. Physical properties, such as sound, humidity, pressure, luminosity, temperature, or chemical concentration, etc. are sensed using these autonomous devices. For further analysis and processing of the data sensed is sent collectively to the base station whose location is static.

These sensor nodes organise themselves into a network to operate more efficiently in terms of both data acquisition and energy consumption. The limited sensor nodes resources such as memory, computational ability, communication bandwidth and energy source are the challenges in network design. Hence the main focus is to select such a routing protocol assisted transmission mechanism that takes into consideration the optimal use of these limited resources. 
There have been numerous routing protocols that have appeared in WSNs. The first low-energy adaptive clustering hierarchy i.e. LEACH [4] was proposed by Heinzelman et al[5]. It is a hierarchical and self-organized cluster-based approach. The area whose information has to be transmitted i.e. the area under monitoring where the sensor nodes are deployed is subdivided randomly into several clusters. Within each cluster $\mathrm{CH}$ (cluster head) is selected which collect data from the associated member nodes in their clusters based on Time Division Multiple Access (TDMA) scheduling. The outcome is transmitted to the base station or sink as a data packet after the removal of redundant data. $\mathrm{CHs}$ are selected through a BS message after a pre-determined period of time. In this paper, effort has been done to optimize the lifetime of the network and the consumption of the energy in WSN by proposing a new clustering protocol using the genetic approach.

The rest of the paper is organized as follows: Section 2 reviews the previous works on clustering approaches. Section 3 consists of the proposed technique using Genetic Algorithm. Section 4 shows simulation results. Section 5 finally describes conclusion.

\section{Related Work}

Many earlier studies contribute in presenting algorithms where the costs, including receiving and transmitting between $\mathrm{CHs}$ and $\mathrm{BS}$, are reduced and the network lifetime is increased. In 2002, Lindsey proposed the PEGASIS protocol, which was an extension of the LEACH algorithm. PEGASIS contribute to the robustness of node failure as compared to LEACH, Pan introduced a two-tiered architecture in which more energy efficiency is provided by hierarchical. Kalpakis [6] and Jin proposed the MLDA (Maximum Lifetime Data gathering Algorithm) to find edge capacities that allows maximum transmission. Dasgupta [6] applied a cluster-based heuristic algorithm which extended MLDA to CMLDA, where nodes are grouped into several pre-defined sized clusters. Bandyopadhyay [7] proposed a multi-level hierarchical clustering algorithm and Cerpa described the Adaptive Self Configuring sensor Networks Topology. An early implementation of GA algorithm is Tur-gut work which applied the GA concept to improve mobile adhoc network clustering.

The proposed algorithm is the same as most of the GA based protocols in that it presents a fitness parameter that decides the destiny of an individual. Jin $[8,1]$, in his work, utilized GA to reduce energy consumption. Simulations show $\mathrm{CH}$ reduction by approximately $10 \%$ of the total number of nodes. Earlier simulations have also shown that cluster-based methods reduce $80 \%$ of the communication distance.

\section{Proposed Algorithm}

The proposed algorithm uses Genetic Algorithm [8] in LEACH to optimize the selection of cluster heads for the arbitrary sensor network. After the cluster heads are selected, the regular nodes are associated with respective nearest cluster heads. Hence, independent clusters are generated in a network. Each normal node can be associated with only one cluster head. Each regular node sends its data to its respective cluster head and the cluster head collects the data from all its associated nodes and sends it to the base station.

In Genetic Algorithm, the principles of natural selection and evolution are used. At each generation, a coded form of a possible solution for the population of individuals is maintained. This is called the chromosome. A chromosome is made up of genes. The various chromosomes are evaluated using a fitness function. The chromosome with the best fitness value is used for generating the next population. After the evaluation step, the population for the next generation is made using selection, crossover and mutation operations. 


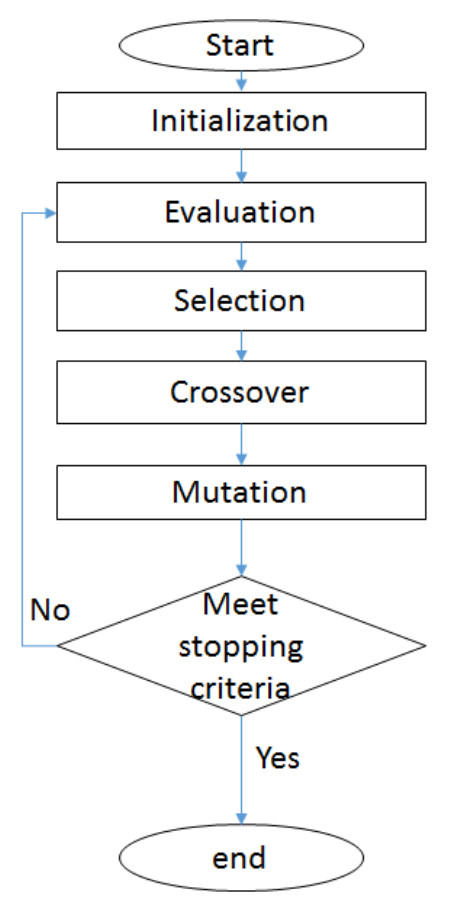

Figure 1. Flowchart for Basic GA

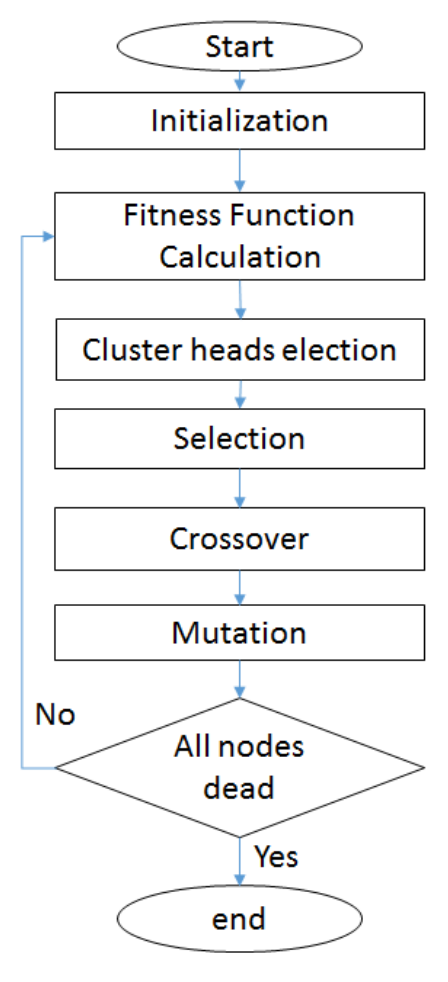

Figure 2. Flowchart for OGA

\section{Population Initialization}

To start the algorithm, an initial population has to be pre-generated. In this paper, the initial population have been generated using a random number algorithm. A random number has been chosen and on the basis of this number it has been checked if the gene in the particular chromosome should be 1 or 0 . In the chromosome, 1 represents a node which is a candidate to be the cluster head. A chromosome defines the possible arrangement for the cluster heads and normal nodes in sensor network. The various positions of the chromosome string represent the genes of the population.

\section{Fitness Function and Cluster Head Selection}

The fitness function is the main part of a Genetic Algorithm. A new fitness function has been designed for the evaluation of the fittest chromosome.

$$
\mathrm{F}(\mathrm{C})=\frac{\operatorname{sumE} * \sin (\mathrm{n} A)}{E c h * D c h}
$$

Ech $=$ sum of energies of candidate cluster heads

Dch $=$ sum of distances of candidate cluster heads

sumE $=$ sum of energies of alive nodes

$\mathrm{nA}=$ number of alive nodes

According to this fitness values, the fittest chromosome is used for the selection of cluster heads in the network for the current generation. The next step is to select the cluster heads from the best chromosome. An optimum number of cluster heads has been selected on the basis of maximum energy values.

After the cluster heads are selected, the normal nodes are associated with their respective cluster heads on the basis of nearest distance. After this step, independent clusters are made. The normal nodes send their data only to their respective cluster head and the cluster heads send the collected data to the base station. 


\section{Selection of Parents from the Current Generation}

This procedure selects two chromosomes from the current generation which will be used to generate an offspring for the next generation. The Tournament Selection Algorithm has been used for this purpose. It selects two chromosomes randomly from the current generation. These two chromosomes are then used as parents in the crossover process for the generation of the offspring.

\section{Crossover}

The two chromosomes selected from the Tournament Selection algorithm undergo crossover operation for the generation of next generation's offspring. Single-point crossover is done between the two parent chromosomes selected. The new population size remains the same as previous generation's population size.

Example:

Chromosome 1: $011101 \mid 011$

Chromosome 2: \begin{tabular}{cccc|ccc}
1 & 0 & 0 & 1 & 1 & 0 & 1
\end{tabular}

\section{Crossover-point}

NewChromosome 1: 01101101

NewChromosome 2: 11001011

\section{Mutation}

Mutation is done to alter some gene values in a chromosome so that genetic variation can be maintained. It occurs during evolution according to a mutation probability already defined by us. The value should be set low to avoid a primary random search.

Example:

Chromosome: $\quad 00110011$

NewChromosome:0 0110110

\section{Simulation}

\section{Simulation Overview}

This work assumes that all sensor nodes are homogeneous and distributed uniformly over the sensor field with limited energy that the links between nodes are symmetric, and that messages from all nodes can reach the BS. The nodes are distributed randomly in a square of size $M \times M$. Extensive simulations were performed on several 100 node networks in a $50 \mathrm{~m} * 50 \mathrm{~m}$ field. The base station was located at $(50 \mathrm{~m}, 50 \mathrm{~m})$ as shown in figure 1 .

Simulations performed in MATLAB show that LEACH using Genetics outperforms the data gathering schemes such as data transmission and multi-hop routing. For implementing the energy efficient data gathering protocol, the cluster head formation was done using Genetic algorithm with simulated annealing.

In every round, optimum number of clusters (5-8) will be formed based on fitness function value for all chromosomes. 


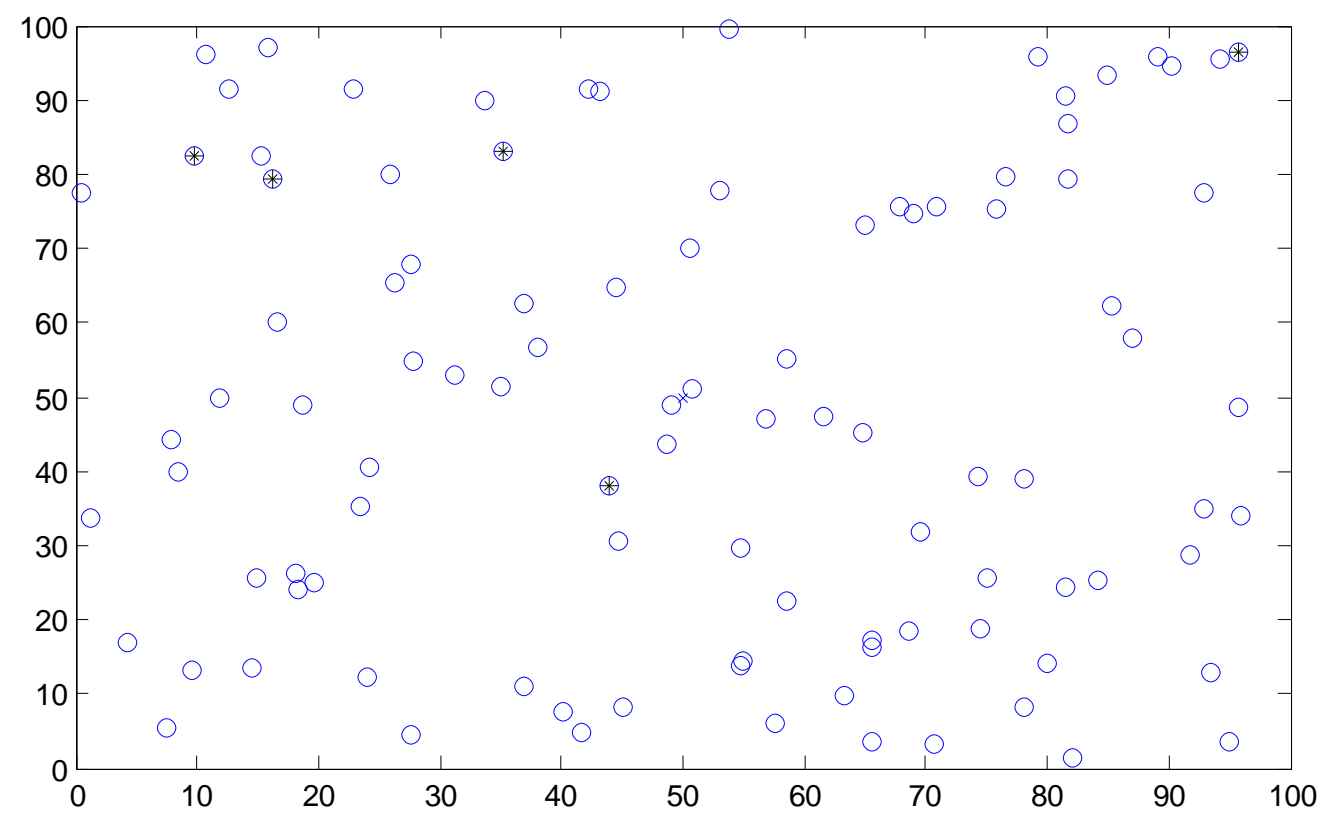

Figure 3. Sensor Network

\section{Simulation Parameters}

Table 1 shows the different simulation parameters including the genetic algorithm used to simulate the environment. The candidate chromosomes can be chosen randomly because this selection does not affect the final results. The network can be functioning as long as the minimum numbers of nodes are alive. Here, an algorithm is used containing fitness function that considers the energy status of candidate cluster head nodes in a chromosome and the distance between candidate $\mathrm{CHs}$ and the $\mathrm{BS}$ where the final individuals provide a cluster formation that uniformly consumes energy. This phenomenon significantly extends the lifetime of the network.

Table 1. Simulation Parameters

\begin{tabular}{|l|l|}
\hline \multicolumn{1}{|c|}{ Parameter } & Value \\
\hline Number of nodes & 100 \\
\hline Optimal Election Probability of a node to become cluster head(p) & 0.1 \\
\hline Initial Energy Eo & \\
\hline Maximum number of rounds rmax & 0.5 \\
\hline Population Size & 2000 \\
\hline Number Of Genes & 5 \\
\hline Crossover Probability & 100 \\
\hline Mutation Probability & 0.8 \\
\hline Tournament Selection Parameter & 0.025 \\
\hline Variable Range & 0.75 \\
\hline Number Of Generations = rmax & 3.0 \\
\hline & 2000 \\
\hline
\end{tabular}




\section{Simulation Results}

Table 2 below describes the values of different parameters obtained after running LEACH using genetics code in MATLAB.

Table 2. Simulation Results for $\mathrm{n}=\mathbf{1 0 0}$

\begin{tabular}{|l|l|l|}
\hline Parameter & OGA & LEACH \\
\hline First_dead & 570 & 378 \\
\hline Half_dead & 895 & 943 \\
\hline Last_dead & 1561 & 1582 \\
\hline $\begin{array}{l}\text { Total Number of rounds } \\
\text { Total Packets transmitted } \\
\begin{array}{l}\text { PACKETS_TO_BS_TILL } \\
\text { ROUND }\end{array}\end{array}$ & 6093 & 2000 \\
\hline
\end{tabular}

Figure 2 gives a comparison of normal LEACH implementation and OGA in terms of 'Number of nodes alive' as per number of rounds occurred. Figure 7 displays a comparison of normal LEACH implementation and OGA in terms of 'Total packets transmited to base station' per round.

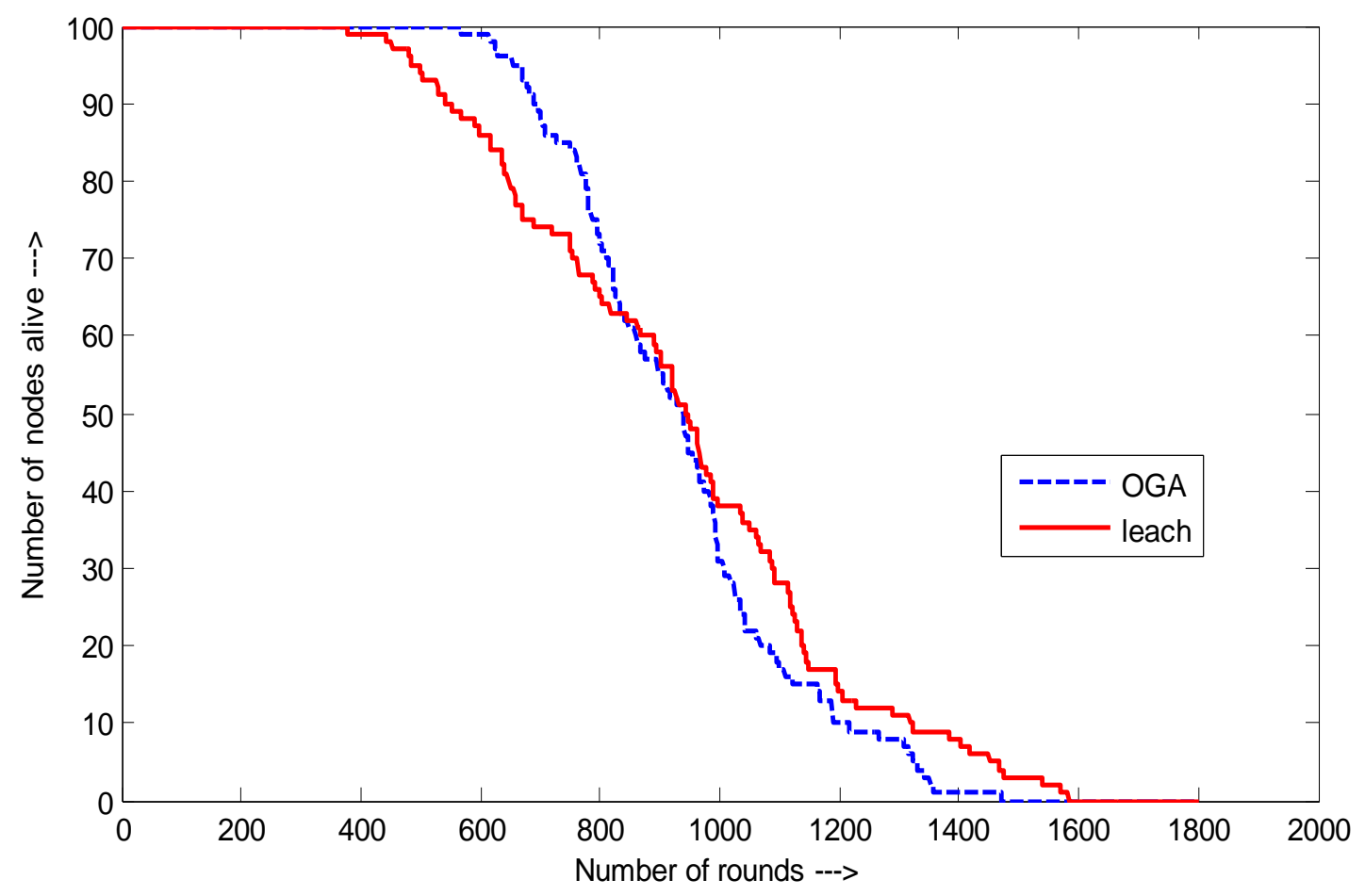

. Figure 4. Number of Nodes Alive, $n=100$ 


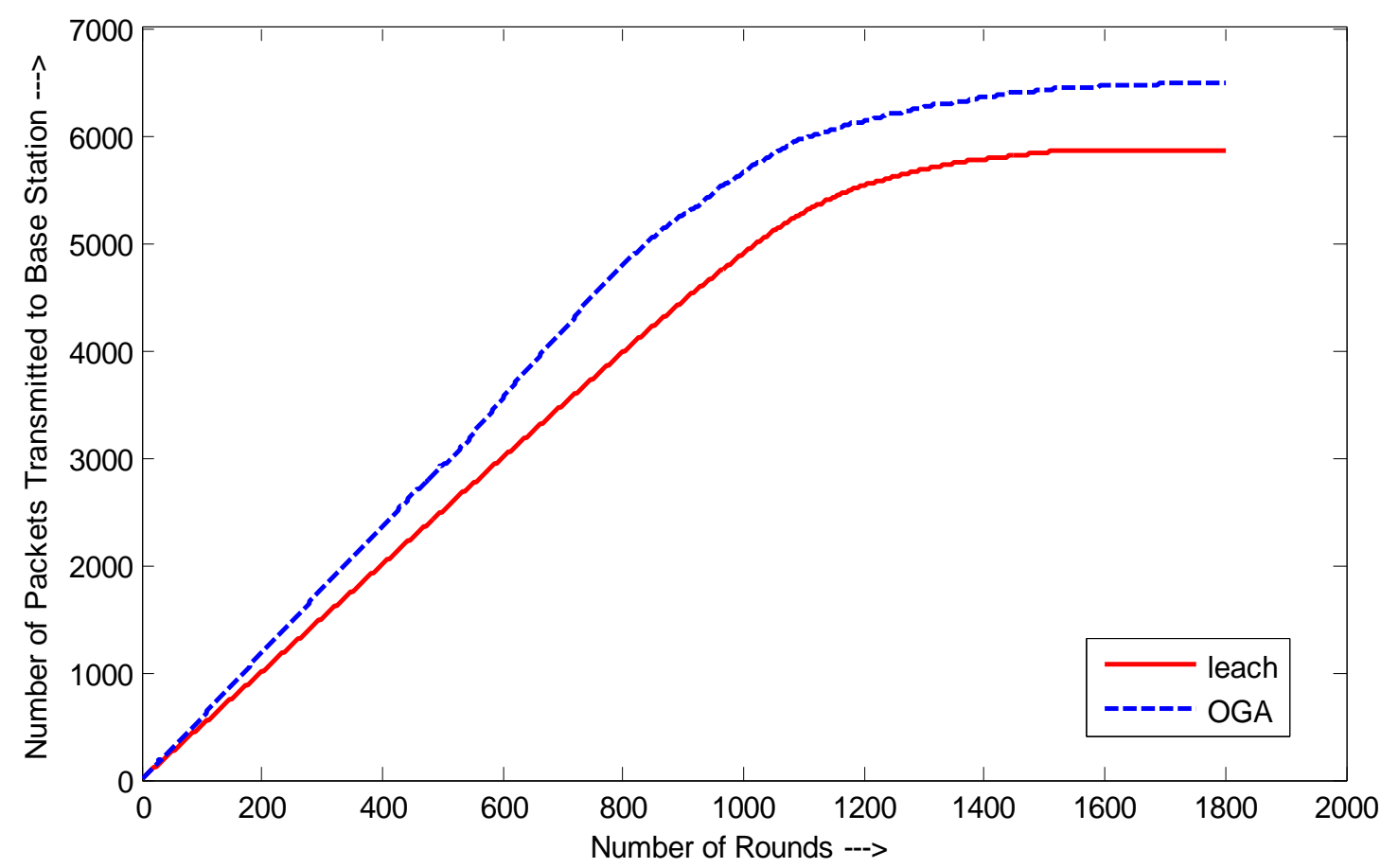

Figure 5. Number of Packets Transmitted, $n=100$

Table 3 gives the simulation results when the number of nodes is increased to 200. It can be seen from the figures 6 and 7 that the OGA performs better even in the 200 nodes network. Even in the 200 node network, the stability period has been increased while the instability period decreased in OGA as compared to LEACH.

Table 3. Simulation Results for $\mathbf{n}=\mathbf{2 0 0}$

\begin{tabular}{|l|l|l|}
\hline Parameter & OGA & LEACH \\
\hline First_dead & 314 & 222 \\
\hline Half_dead & 945 & 982 \\
\hline Last_dead & 1562 & 1655 \\
\hline Total Number of rounds & 2000 & 2000 \\
\hline $\begin{array}{l}\text { Total Packets transmitted } \\
\text { PACKETS_TO_BS_TILL } \\
\text { ROUND }\end{array}$ & 8890 & 6822 \\
\hline
\end{tabular}




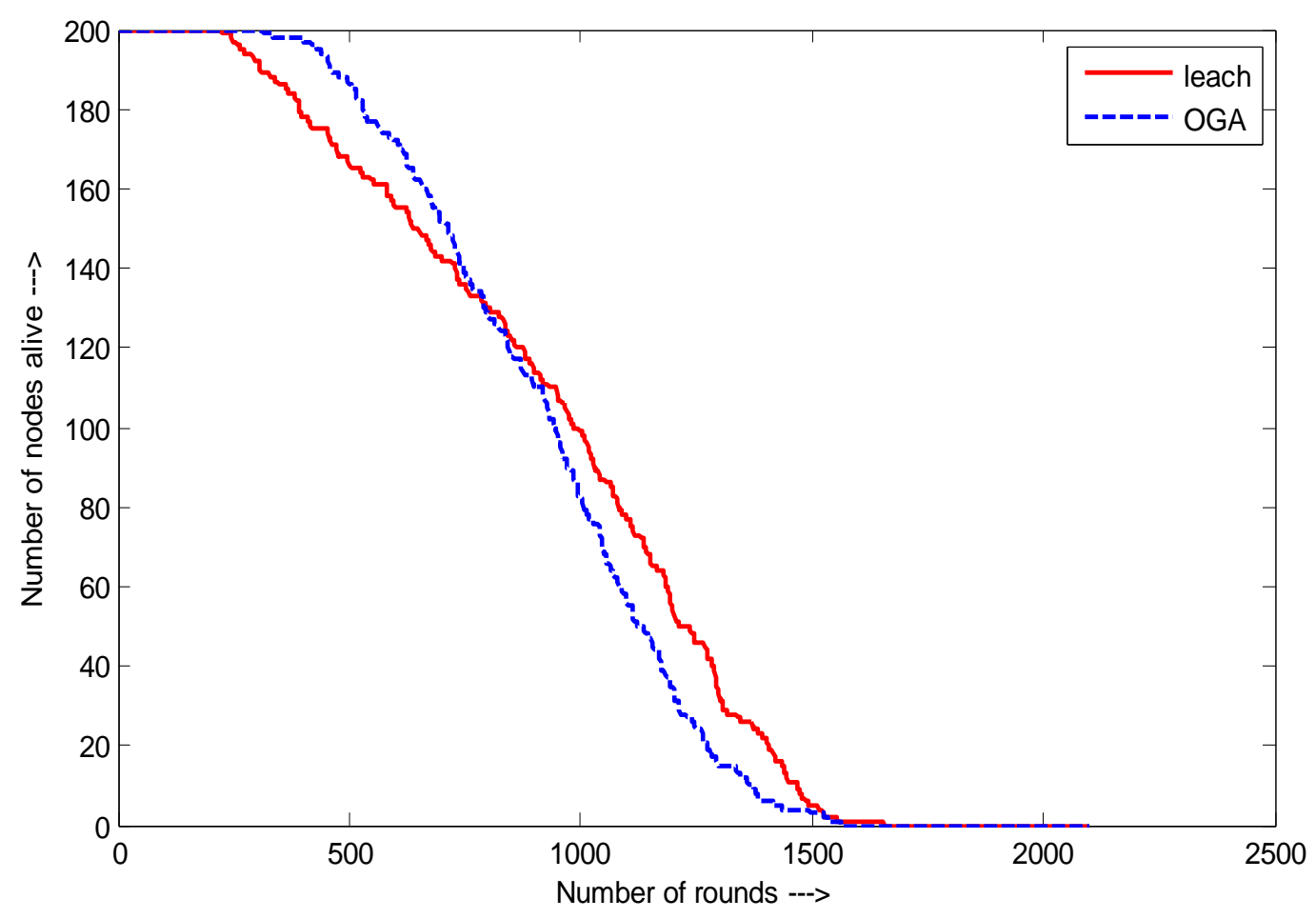

Figure 6. Number of Nodes Alive, $n=200$

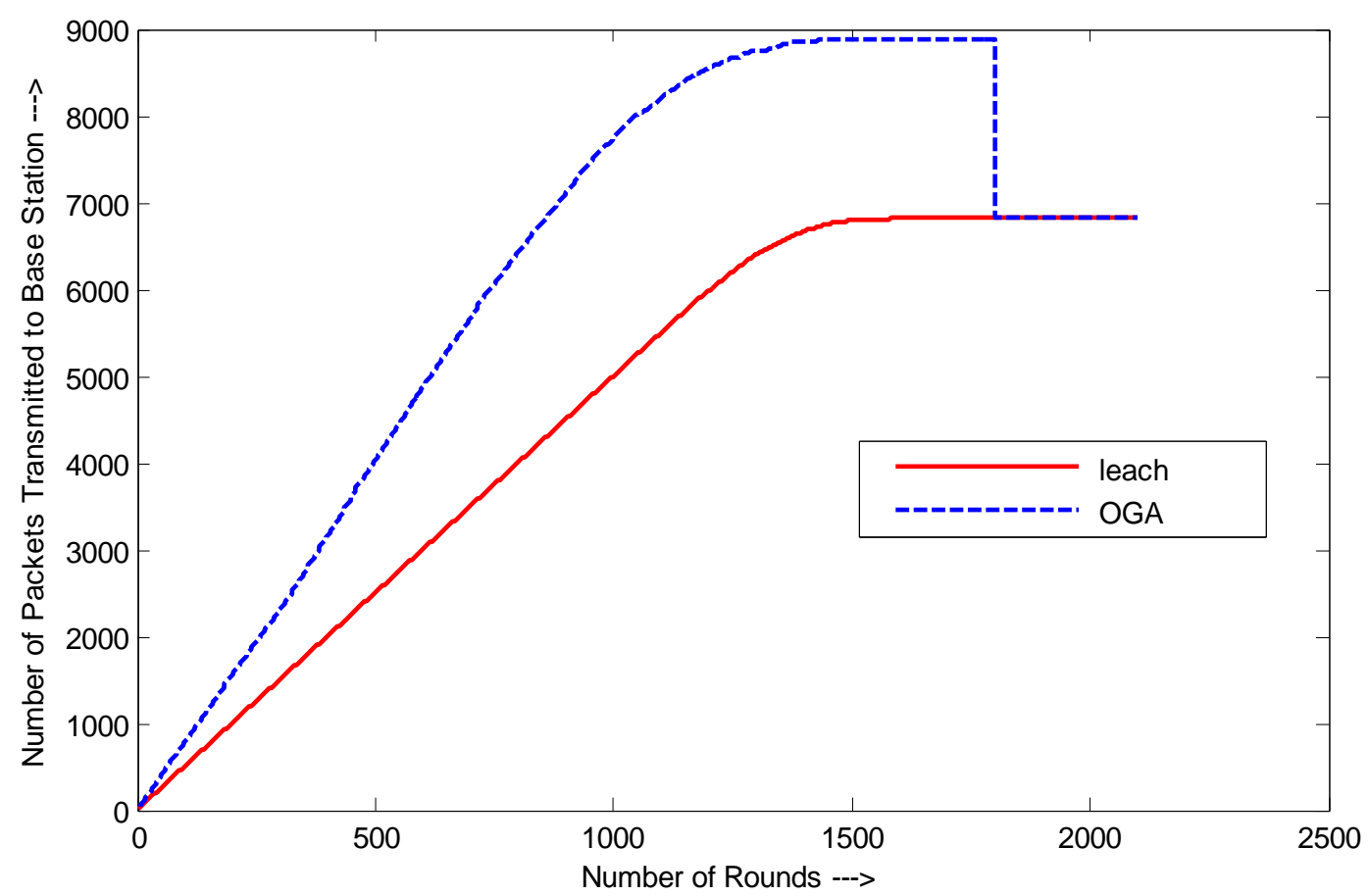

Figure 7. Number of Packets Transmitted, $n=200$ 


\section{Conclusion and Future Work}

A WSN consists of a number of small sensor nodes used to entirely cover an environment; hence, the sensor nodes should be low cost, low power and have limited energy use. These nodes can communicate to each other across a short distance. WSNs may be deployed either randomly or deterministically, depending upon the application. Cluster based approaches are appropriate for monitoring applications that require a continuous stream of sensor data; thus, routing protocols are applied to lower the cost of delivering a data packet on time. In this paper, a clustering algorithm has been proposed for routing wireless sensor networks based on genetic algorithm. The proposed algorithm begins by randomly selecting nodes in a network to be cluster-heads. By adjusting clusterheads based on fitness function, the proposed algorithm is able to find an appropriate number of cluster-heads and their locations. The proposed algorithm takes different parameters into consideration to increase the network lifetime. The main parameters considered for evaluating fitness function for selecting next population are - energy of candidate cluster head nodes and the distance of candidate cluster head nodes to base station. The results of the simulations show the effectiveness of the proposed mechanism. As proved by simulation results, the proposed algorithm has prolonged network lifetime by increasing the number of rounds at which half the number of nodes die and the number of round at which last node dies inside the network. This algorithm is also increasing the number of packets transmitted as compared to that in LEACH by a factor of 10 .

The future work will be to maximize network stability for heterogeneous networks. Due to the drawbacks of LEACH, many protocols have been emerged to solve these problems [21-22]. However, various surveys have displayed [23-24] that more work is still needed to find more efficient, scalable and robust clustering scheme to enhance energy consumption and prolonging networks lifetime in small and large WSNs.

\section{References}

[1] K. SIM, J. Choi, K. Kwon and J. Lee, "Energy Efficient Cluster Header Selection Algorithm in WSN", in the proceedings off IEEE international Conference on Complex, Intelligent and Software Intensive Systems, (2009).

[2] S. Zhu, W. Wang and C. V. Ravishankar, "PERT: A new power-efficient real-time packet delivery scheme for sensor networks", International Journal of Sensor Networks, vol.3, no.4, (2008), pp.237-251.

[3] Woo and D. E. Culler, "Evaluation of E_cient Link Reliability Estimators for Low-power Wireless Networks", Computer Science Division, University of California, (2003).

[4] J.-L. Liu and C. V. Ravishankar, "LEACH-GA: Genetic Algorithm-Based Energy-Efficient Adaptive Clustering Protocol for Wireless Sensor Networks", International Journal of Machine Learning and Computing, vol.1, no.1, (2011).

[5] W. R. Heinzelman, A. Chandrakasan and H. Balakrishnan, "Energy efficient communication protocol for wireless microsensor networks", In Proceedings of the Hawaii International Conference on System Sciences, (2000).

[6] Dasgupta, Koustuv, Kalpakis, Konstantinos, and Namjoshi, Parag, "An efficient clustering-based heuristic for data gathering and aggregation in sensor networks", In IEEE Wireless Communications and Networking Conference, (2003).

[7] S. Bandyopadhyay and E. J. Coyle, "An energy efficient hierarchical clustering algorithm for wireless sensor networks", In Proceedings of the IEEE Conference on Computer Communications (INFOCOM), (2003).

[8] S. Jin, M. Zhou, S. Annie, "Sensor Network Optimization Using a Genetic Algorithm", Wu School of EECS University of Central Florida Orlando, FL 32816.

[9] S. Hussain, A. W. Matin and O. Islam, "Genetic Algorithm for Hierarchical Wireless Sensor Networks", Jodrey School of Computer Science, Journal of networks, Acadia University, Wolfville, Nova Scotia, Canada, vol.2, no.5, (2007).

[10] D. S. Rao, B. J. M. R. Kumar, "Performance Evaluation of Genetic Based Dynamic Clustering Algorithm over LEACH Algorithm for Wireless Sensor Networks", International Journal of Soft Computing and Engineering, ISSN: 2231-2307, vol.1, no.4, (2011).

[11] A. Chakraborty, S. K. Mitra and M. K. Naskar, "A Genetic Algorithm inspired Routing Protocol for Wireless Sensor Networks", International journal of computational intelligence theory and practice, Dept. of computer science and engineering, Jadavpur University. 
[12] I. F. Akyildiz, W. Su, Y. Sankarasubramaniam and E. Cayirci, "Wireless sensor networks: A survey", Computer Networks, vol.38, no.4, (2002), pp.393-422.

[13] J. M. Johnson and Y. R. Samii, "Genetic Algorithm Optimization of Wireless Communication Networks", Proceedings for Antennas and Propagation Society International Symposium, AP-S. Digest, (1995).

[14] H. Sajid, Islam, Obidul, Matin and A. Wasey, "Genetic algorithm for energy efficient clusters in wireless sensor networks", In Proceedings of the 4th International Conference on Information Technology: New Generations, IEEE Computer Society, (2007).

[15] Islam, Obidul and Hussain, Sajid, "Effect of layers on simulation of wireless sensor networks", In Proceedings of the Third International Conference on Wireless and Mobile Communications (ICWMC). IEEE Computer Society, (2007).

[16] G. Smaragdakis, I. Matta and A. Bestavros, "SEP: A Stable Election Protocol for clustered heterogeneous wireless sensor networks, Second International Workshop on Sensor and Actor Network Protocols and Applications, (2004).

[17] M. Wahde and D. Sandberg, "An elementary introduction to Matlab programming for stochastic optimization", (2010).

[18] O. Tan, Hu"seyin and I. Ko"rpeog lu, "Power efficient data gathering and aggregation in wireless sensor networks", SIGMOD Rec., vol.32, no.4, (2003), pp.66-71.

[19] V. Erramilli, I. Matta and A. Bestavros, "On the interaction between data aggregation and topology control in wireless sensor networks", In Proceedings of Secon, (2004), pp.557-565.

[20] K, P. Ferentinos, T. A. Tsiligiridis and K. G. Arvanitis, "Energy optimization of wirless sensor networks for environmental measurements", In Proceedings of the International Conference on Computational Intelligence for Measurment Systems and Applicatons, (2005).

[21] S. Hussain and A. W. Matin, "Base station assisted hierarchical cluster-based routing", In IEEE/ACM International Conference on Wireless and Mobile communications Networks, (2006).

[22] K. Yang, Y. Wu and H. Zhou, "Research of optimal energy consumption model in wirelss sensor network", In 2010 2nd International Conference on Computer Engineering and Technology, (2010); Chengdu, China.

[23] P. T. V. Bhuvaneswari and V. Vaidehi, "Enhancement techniques incorporated in LEACH- a survey", Indian Journal of Science and Technology, vol.2, no.5, (2009), pp.36-44.

[24] A. Abbasi and M. Younis, "A survey on clustering algorithms for wireless sensor networks", Computer Communications, vol.30, (2007), pp.2826-2841. 